\title{
FGF21 Mediates Mesenchymal Stem Cell Senescence via Regulation of Mitochondrial Dynamics
}

\author{
Xin Li, ${ }^{1}$ Yimei Hong, ${ }^{1,2}$ Haiwei He, ${ }^{1,2}$ Guojun Jiang, ${ }^{3}$ Wei You, ${ }^{1}$ Xiaoting Liang, ${ }^{4}$ \\ Qingling Fu, ${ }^{5}$ Shuo Han, ${ }^{6}$ Qizhou Lian, ${ }^{6}$ and Yuelin Zhang $\mathbb{C I}^{1,3}$ \\ ${ }^{1}$ Department of Emergency Medicine, Department of Emergency and Critical Care Medicine, Guangdong Provincial \\ People's Hospital, Guangdong Academy of Medical Sciences, Guangzhou, Guangdong 510080, China \\ ${ }^{2}$ School of Medicine, South China University of Technology, Guangzhou, Guangdong 510515, China \\ ${ }^{3}$ Faculty of Pharmacy, Bengbu Medical College, Bengbu, Anhui 233000, China \\ ${ }^{4}$ Clinical Translational Medical Research Center, Shanghai East Hospital, Tongji University School of Medicine, \\ Shanghai 200120, China \\ ${ }^{5}$ Otorhinolaryngology Hospital, The First Affiliated Hospital, Sun Yat-sen University, Guangzhou, Guangdong 510080, China \\ ${ }^{6}$ Department of Medicine, Li Ka Shing Faculty of Medicine, The University of Hong Kong, Hong Kong
}

Correspondence should be addressed to Yuelin Zhang; zhangyuelin1999@163.com

Received 28 January 2019; Accepted 26 March 2019; Published 17 April 2019

Academic Editor: Nady Braidy

Copyright (C) 2019 Xin Li et al. This is an open access article distributed under the Creative Commons Attribution License, which permits unrestricted use, distribution, and reproduction in any medium, provided the original work is properly cited.

\begin{abstract}
Mesenchymal stem cell- (MSC-) based therapy is a novel strategy in regenerative medicine. The functional and regenerative capacities of MSCs decline with senescence. Nonetheless, the potential mechanisms that underlie their senescence are not fully understood. This study was aimed at exploring the potential mechanisms of fibroblast growth factor 21 (FGF21) in the regulation of MSC senescence. The senescence of MSCs was determined by senescence-associated $\beta$-galactosidase (SA- $\beta$-gal) staining. The morphology and the level of mitochondrial reactive oxygen species (ROS) of MSCs were assessed by MitoTracker and Mito-Sox staining, respectively. The expression of FGF21 and mitochondrial dynamics-related proteins was detected by Western blotting. As MSCs were expanded in vitro, the expression of FGF21 decreased. Depletion of FGF21 enhanced production of mitochondrial reactive oxidative species (ROS) and increased the senescence of early-passage MSCs whereas inhibition of ROS abolished these effects. The senescent MSCs exhibited increased mitochondrial fusion and decreased mitochondrial fission. Treatment of early-passage MSCs with FGF21 siRNA enhanced mitochondrial fusion and reduced mitochondrial fission. Moreover, treatment of mitofusin2- (Mfn2-) siRNA inhibited depletion of FGF21-induced MSC senescence. Furthermore, we demonstrated that depletion of FGF21-induced mitochondrial fusion was regulated by the AMPK signaling pathway. Treatment with an AMPK activator, AICAR, abrogated the depletion of FGF21-induced senescence of MSCs by inhibiting mitochondrial fusion. Compared with MSCs isolated from young donors, those derived from aged donors showed a lower level of FGF21 and a higher level of senescent activity. Furthermore, overexpression of FGF21 in aged MSCs inhibited senescence. Our study shows that FGF21, via the AMPK signaling pathway, regulates the senescence of MSCs by mediating mitochondrial dynamics. Targeting FGF21 might represent a novel strategy to improve the quality and quantity of MSCs.
\end{abstract}

\section{Introduction}

Over the past decades, mesenchymal stem cell- (MSC-) based therapy has emerged as a promising tool for regenerative medicine due to its self-renewal, multiple-lineage differentiation, and immunomodulatory capacity. Although MSCs can be easily obtained from several tissues in the human body, they need to be expanded in vitro for several weeks to obtain an adequate amount for clinical trials $[1,2]$. Nonetheless, MSCs can undergo only limited cell divisions and prolonged expansion inevitably leads to replicative senescence $[3,4]$. This replicative senescence remarkably diminishes their functional and regenerative capacity, thus reducing their therapeutic efficacy [5-7]. The potential mechanisms that 
underlie the cellular senescence of MSCs remain largely unknown.

Fibroblast growth factor 21 (FGF21), a member of the FGF family, is an endocrine factor that primarily mediates glucose and lipid metabolism. Recently, accumulating evidence has demonstrated that FGF21 plays a critical role in the antiaging process. FGF21 treatment can ameliorate a variety of age-related metabolic diseases, and transgenic mice that overexpress FGF21 have an extended lifespan [8, 9]. Furthermore, FGF21 delays the replicative senescence of endothelial cells by regulating the level of SIRT1 [10]. FGF21 represses angiotensin-induced cellular senescence of human brain vascular smooth muscle cells by regulating mitochondrial biogenesis [11]. Whether and how FGF21 regulates the cellular senescence of MSCs have not been determined.

Reactive oxidative species (ROS) is one of the wellestablished senescence-triggering mechanisms. A pesticide mixture induces cellular senescence of MSCs by upregulating ROS generation, thus impairing their differentiation capacity [12]. ROS-induced suppression of c-Maf leads to the replicative senescence of MSCs, increasing adipogenic differentiation and reducing osteogenic differentiation [13]. To the best of our knowledge, mitochondria are the major source of ROS. Mitochondria constantly undergo fission and fusion and form a dynamic network to maintain cell function [14]. Mitochondrial fission is mainly regulated by dynaminrelated protein 1 (Drp1) and fission-1 (Fis1), and mitochondrial fusion is regulated by Mitofusin1 (Mfn1) and Mitofu$\sin 2$ (Mfn2) and optic atrophy protein 1 (OPA1). Phosphorylation of Drp1 at Ser616 (p-Drp1 Ser616) increases Drp1 translocation to mitochondria and therefore induces mitochondrial fission [15]. It is well documented that the mitochondrial structure is closely associated with ROS generation and disruption of mitochondrial dynamics results in ROS production with consequent cell injury and cellular senescence [16-20]. Nonetheless, whether FGF21 mediates MSC senescence by regulating mitochondrial dynamics remains to be elucidated.

Given these findings, we hypothesized that FGF21 mediates MSC senescence via regulation of mitochondrial dynamics and ROS production. We show that depletion of FGF21induced mitochondrial fusion and ROS overproduction lead to senescence of MSCs. Furthermore, FGF21-regulated mitochondrial dynamics are involved in the AMPK signaling pathway.

\section{Materials and Methods}

2.1. Cell Culture. Bone marrow (BM) MSCs were cultured with DMEM/high glucose (Gibco) plus 10\% FBS (Life Technologies, 16000), $0.1 \mathrm{mM} 2$-mercaptoethanol (Life Technologies, 21985023), NEAA (Life Technologies, 11140050), penicillin-streptomycin (Life Technologies, 15140122), $5 \mathrm{ng} / \mathrm{mL}$ EGF (PeProTech, AF-100-15), and $5 \mathrm{ng} / \mathrm{mL}$ FGF (PeProTech, 100-18B) at $37^{\circ} \mathrm{C}$ in a $5 \% \mathrm{CO}_{2}$ incubator. MSCs were passaged at a ratio of $1: 4$ when they reached $80 \%-90 \%$ confluence. Human young MSCs and aged MSCs were harvested from volunteer donors at Shanghai East Hospital as previously described [21]. The procedure was approved by the Ethics Committee of Shanghai East Hospital, and written informed consent was obtained from all the volunteers. The passage 4-5 of young MSCs and aged MSCs was used in the current study. Informed consent was obtained from all volunteers. According to age, a total of twenty volunteers were divided into young (young, 18-25 years old) or aged group (aged, 65-80 years old).

2.2. Lentiviral Construct Packaging and Infection. Two lentivirus-based recombinant plasmids were constructed. One was inserted by FGF21; the other was inserted by the same backbone as control. The lentiviruses were packaged by transfecting 293T cells using the lentiviral packaging system that comprised the recombinant lentiviral transfer FGF21 plasmid, packaging (GAG/Pol and REV) plasmids, and envelope (VSV-G) plasmid. Following 48 hours of culture, the supernatant of transfecting 293T cells was collected, concentrated, and tittered. Subsequently, the virus was used to infect BM-MSCs. Finally, gene transduction efficiency was evaluated by Western blotting.

2.3. Senescence-Associated $\beta$-Galactosidase (SA- $\beta$-Gal) Staining. SA- $\beta$-gal staining was performed according to the manufacturer's protocol (Cell Signaling Technology). Briefly, MSCs were washed with PBS and fixed using the fixative solution for half an hour at room temperature and then incubated at $37^{\circ} \mathrm{C}$ overnight with the SA- $\beta$-gal staining solution. The senescent MSCs stained with blue were photographed. The percentage was calculated from five different view fields of each sample in three independent experiments.

2.4. Small-Interfering RNA (siRNA) Silencing. FGF21 siRNA (Santa Cruz, sc-39484), Mfn2 siRNA (Santa Cruz, SC43928), and control siRNA were used to transfect MSCs using a Lipofectamine RNAiMAX Reagent Kit (Invitrogen, 13778-075) at a standardized MOI (multiplicity of infection) of 5 according to the protocol. Seventy-two hours after FGF21 siRNA and Mfn2 siRNA transfection, MSCs were harvested and the silencing efficiency evaluated by Western blotting.

2.5. ELISA. The conditioned medium of MSCs at passage 4 and passage 12 was collected, respectively. In brief, a total of $5 \times 10^{6}$ MSCs were plated on a $10 \mathrm{~cm}$ culture plate. Twenty-four hours later, the culture medium was changed with $10 \mathrm{~mL}$ of serum- and antibiotic-free DMEM. After a further 24 hours, the supernatant was collected gently, filtered through a $0.22 \mu \mathrm{m}$ filter. The concentration of FGF21 in the conditioned medium was determined by FGF-21 ELISA Kit (DF2100, R\&D Systems).

2.6. Mdivi-1 Treatment. MSCs were incubated with mitochondrial fission protein Drp1 inhibitor Mdivi-1 (SC215291, $10 \mu \mathrm{M}$ ) for 24 hours when MSCs reached 60 70\% confluence and were then cultured for a further 48 hours and harvested for the following experiments.

2.7. Measurement of Mitochondrial ROS. Mitochondrial ROS in MSCs were determined by Mito-Sox staining. Briefly, MSCs were cultured in 24-well plates with glass coverslips 
with different treatments. Next, MSCs were incubated with $5 \mu \mathrm{M}$ Mito-Sox (Invitrogen, M36008) for $15 \mathrm{~min}$ at $37^{\circ} \mathrm{C}$ in the dark. Finally, the sample was randomly photographed and the fluorescence intensity analyzed from five different view fields of each group using ImageJ software in three independent experiments.

2.8. Western Blotting. The proteins of MSCs with different treatments were extracted and the concentrations measured. A total of $20 \mu \mathrm{g}$ protein from each sample was loaded, separated by SDS/PAGE, and then transferred to a PVDF membrane. Next, after blocking with 5\% fat-free milk in TBST, the membrane was incubated at $4^{\circ} \mathrm{C}$ overnight with the following antibodies: anti-FGF21 (Abcam, ab171941), antip53 (Abcam, ab26), anti-p21 (Abcam, ab109199), anti-Ki67 (Abcam, ab15580), anti-p-Drp1 ser616 (Invitrogen, PA5-64821), anti-Drp1 (Invitrogen, PA5-20176), anti-Mfn2 (Abcam, ab124773), and GAPDH (Santa Cruz, SC-137179). The membrane was washed with TBST and incubated with horseradish peroxidase-conjugated secondary antibodies (1:5000; Santa Cruz) at room temperature for one hour and then proceeded to development.

2.9. MitoTracker Staining. The morphology of MSCs was examined by MitoTracker Green FM (Invitrogen, Carlsbad, CA, USA). MSCs given different treatments were incubated for $30 \mathrm{~min}$ with DMEM supplemented with $20 \mathrm{nM}$ MitoTracker Green FM. After washing with PBS three times, cells were mounted with DAPI and photographed using a confocal microscope.

2.10. Statistical Analysis. All data are presented as the mean \pm SEM. Statistical analyses were performed using Prism 5.04 software (GraphPad Software for Windows, San Diego, CA, USA). Comparison between two groups was analyzed by unpaired Student's $t$-test and comparison between multiple groups by one-way ANOVA followed by the Bonferroni test. A $p$ value $<0.05$ was considered statistically significant.

\section{Results}

3.1. Expansion of MSCs Induces Cellular Senescence In Vitro. We first examined the morphology of MSCs at different passages under light microscopy. MSCs at passage 4 (P4) showed a healthy spindle shape but an enlarged and flattened shape in passage 8 (P8) and in passage 12 (P12) (Figure 1(a), i). Compared with MSCs in P4, the cell size was significantly increased in P8 and P12 (Figure 1(a), ii). Next, the senescence of MSCs at different passages was examined using SA- $\beta$-gal staining. The percentage of SA- $\beta$-gal-positive cells was greatly enhanced in P8 and P12 compared with P4 cells (Figure 1(b), i and ii). To further validate our observation, Ki-67 staining was performed to examine the proliferative capacity of MSCs at different passages. There was a significant decrease in the percentage of Ki-67-positive MSCs from P4 to P12 (Figure 1(c), i and ii). We also examined the level of cellular senescence-related proteins p53 and p21. Levels of both were markedly elevated in P8 and P12 BM-MSCs compared with those in P4 (Figure $1(\mathrm{~d})$ ). We conclude that BM-
MSCs can develop a senescent phenotype during consecutive passaging expansion.

3.2. Depletion of FGF21 Induces Cellular Senescence of MSCs. To determine the effects of FGF21 on cellular senescence of MSCs, we examined the expression level of FGF21 at different passages. Western blotting showed that FGF21 was gradually reduced in a passage-dependent manner, suggesting that FGF21 may be involved in regulating the senescence of MSCs (Figure 2(a), i and ii). Furthermore, compared with P4 BMMSCs, the concentration of FGF21 in the conditioned medium at P12 BM-MSCs was greatly reduced (128.3 \pm 7.8 $\mathrm{pg} / \mathrm{mL}$ vs. $50.7 \pm 5.9 \mathrm{pg} / \mathrm{mL}$ ), indicating that MSCs secrete FGF21 and the concentration declines during consecutive passaging expansion. To verify whether decreased FGF21 is responsible for inducing senescence of MSCs, we treated P4 BM-MSCs with FGF21 siRNA. We found that FGF21 siRNA treatment greatly reduced the protein level of FGF21 but upregulated the p53 and p21 protein level in P4 BM-MSCs (Figure 2(b), i and ii). FGF21 siRNA treatment also markedly enhanced SA- $\beta$-gal-positivity in P4 BM-MSCs (Figure 2(c), i and ii). Furthermore, Ki-67 staining revealed that the number of Ki-67-positive cells was significantly reduced in FGF21 siRNA-treated P4 BM-MSCs compared with control BMMSCs (Figure 2(d), i and ii). Taken together, these results reveal that knockdown of FGF21 induces cellular senescence in early-passage BM-MSCs.

3.3. FGF21 Knockdown Induces Senescence via ROS Generation. It is well established that ROS from mitochondria plays a very important role in regulating cellular senescence. We therefore used Mito-Sox staining to detect ROS production at different passages of BM-MSCs. As shown in Figure 3(a), the ROS level was significantly enhanced in P8 and P12 BM-MSCs compared with that in P4 (Figure 3(a), i and ii), suggesting that ROS may be involved in the regulation of MSC senescence. FGF21 siRNA treatment markedly enhanced ROS generation in P4 BM-MSCs (Figure 3(b), i and ii). Nonetheless, treatment with MitoTEMPO, a mitochondrial ROS inhibitor, significantly inhibited FGF21 siRNA-induced ROS generation in P4 BM-MSCs (Figure 3(b), i and ii). More importantly, MitoTEMPO treatment also dramatically reduced FGF21 siRNAinduced senescence of P4 BM-MSCs (Figure 3(c), i and ii)). Collectively, our results reveal that FGF21 knockdown induces senescence of MSCs by increasing ROS generation.

3.4. FGF21 Knockdown Induces ROS Generation via Regulation of Mitochondrial Dynamics. Accumulating evidence has demonstrated that mitochondrial fusion and fission are closely related to ROS generation. We first examined the mitochondrial morphology of BM-MSCs during P4 and P12. MitoTracker green staining demonstrated small tubular mitochondria in P4 BM-MSCs but large tubular mitochondria in P12 BM-MSCs, indicating that mitochondrial fusion may contribute to cellular senescence (Figure 4(a)). Western blotting showed that compared with P4 BM-MSCs, Mfn2 was greatly enhanced whereas p-Drp1 was markedly reduced in P12 BM-MSCs (Figure 4(b), i and ii). Furthermore, no significant difference of the protein level 

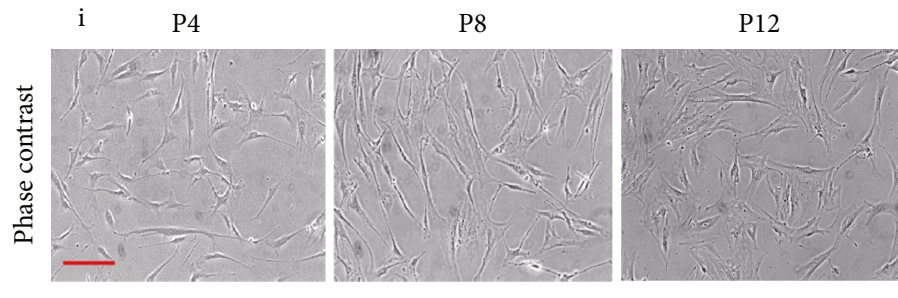

(a)
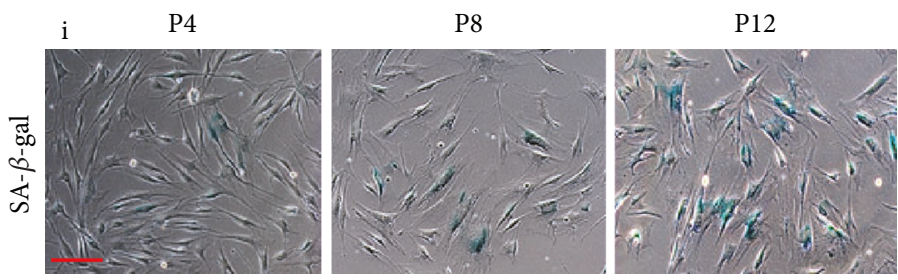

(b)
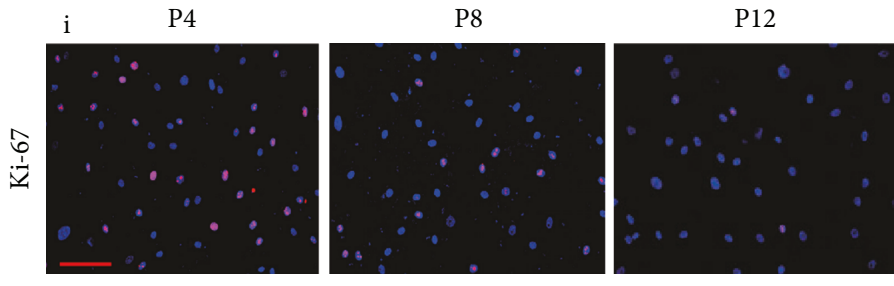

(c)
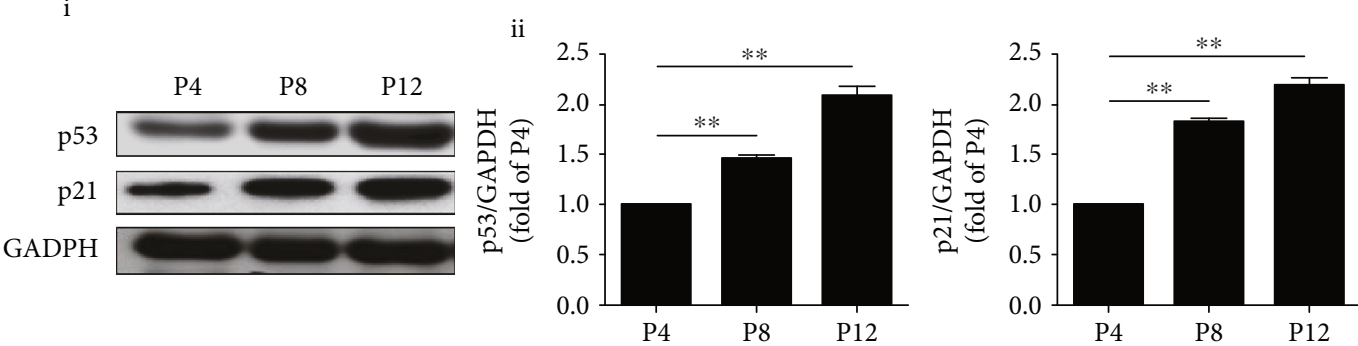

(d)
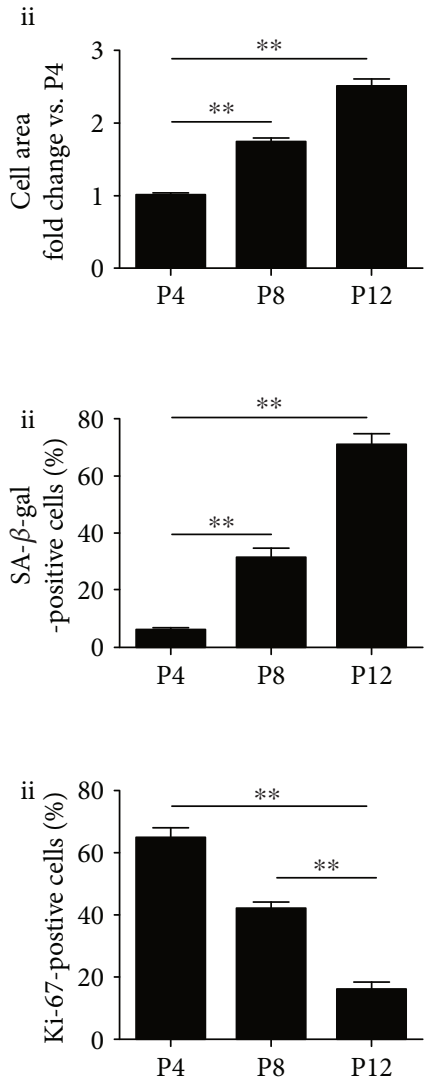

FIgURE 1: Characterization of BM-MSCs replicative senescence. (a) Representative images of BM-MSC morphology under light microscopy at passage 4 (P4), passage 8 (P8) and passage 12 (P12) (i). The cell size of MSCs was calculated at P4, P8, and P12 (ii). (b) Representative images of SA- $\beta$-gal staining in BM-MSCs at P4, P8, and P12 (i). The SA- $\beta$-gal-positive cells at P4, P8, and P12 were calculated and presented as percentage of the total cells (ii). (c) Representative images of Ki-67 staining in BM-MSCs at P4, P8, and P12 (i). The Ki-67positive cells at P4, P8, and P12 were calculated and presented as percentage of the total cells (ii). (d) Western blotting and quantitative analysis of the level of p53 and p21 proteins in BM-MSCs at P4, P8, and P12 (i, ii). Data are expressed as mean \pm SEM with $n=3$ per group. ${ }^{* *} p<0.01$. Scale bar $=100 \mu \mathrm{m}$.

of Fis1, Mfn1, and OPA1 was observed between P4 BMMSCs and P12 BM-MSCs (Figure S1). Next, we treated P4 BM-MSCs with Mdivi-1 for 24 hours and cultured them for a further 48 hours. Western blotting showed that Mdivi-1 treatment enhanced the protein level of Mfn2 and reduced the level of p-Drp1, suggesting the occurrence of mitochondrial fusion (Figure S2A-i, ii). Furthermore, Mdivi-1 treatment significantly increased mitochondrial ROS generation (Figure S2B-i, ii) and enhanced SA- $\beta$-gal positivity of P4 BM-MSCs (Figure S2C-i, ii), indicating that mitochondrial fusion induces senescence of BM-MSCs. To further illustrate the relationship between FGF21 and mitochondrial dynamics, P4 BM-MSCs were transfected with FGF21 siRNA. Silencing FGF21 with FGF21 siRNA elevated the level of Mfn2 and decreased the level of pDrp1 (Figure 4(c)). Meanwhile, ROS generation and senescent activity were enhanced in FGF21 siRNA-treated P4 BM-MSCs (Figures 4(d) and 4(e)). On the contrary, Mfn2 siRNA treatment attenuated the increased expression of Mfn2 and decreased the expression of p-Drp1 in FGF21 siRNA-treated P4 BM-MSCs (Figure 4(c)). Silencing Mfn2 also abrogated FGF21 siRNA-induced ROS generation and 


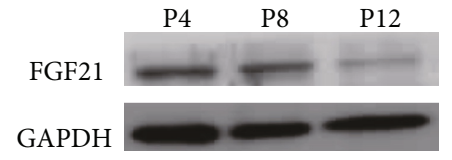

GAPDH

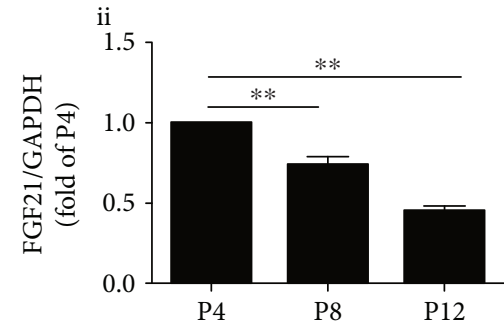

(a)
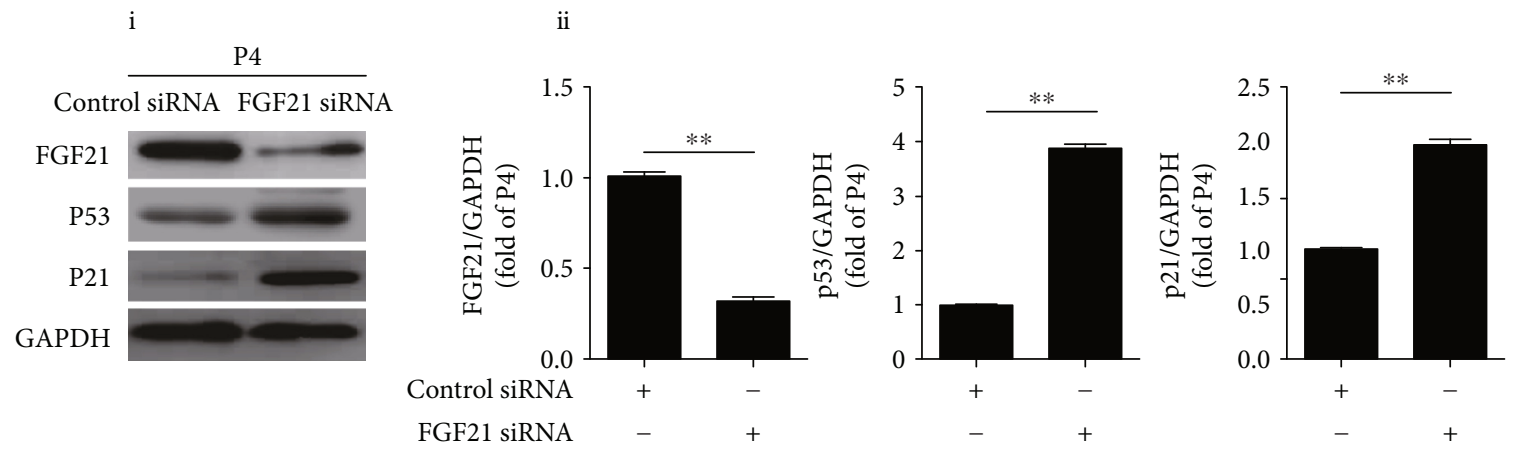

(b)
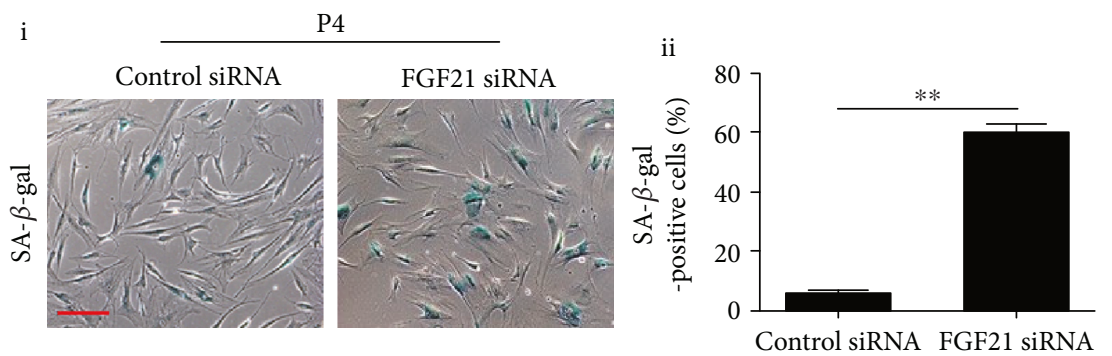

(c)
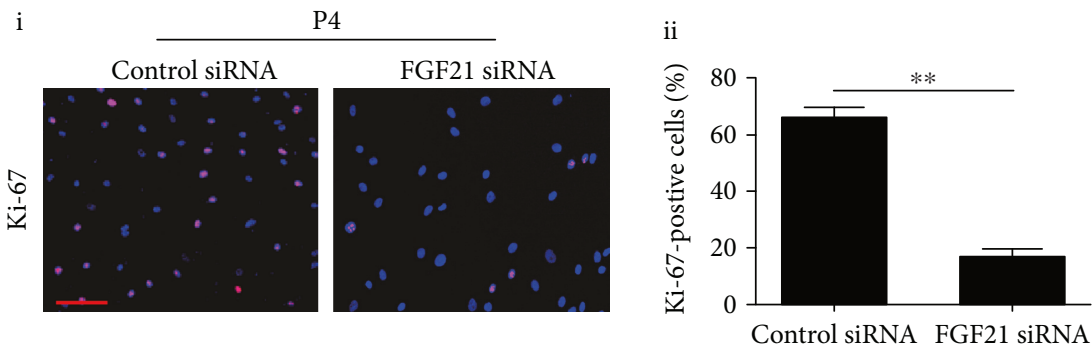

(d)

FIGURE 2: FGF21 mediates the replicative senescence of BM-MSCs. (a) Western blotting and quantitative analysis of the level of FGF21 protein in BM-MSCs at P4, P8, and P12 (i, ii). (b) Western blotting and quantitative analysis of the level of FGF21, p53, and p21 proteins in control siRNA or FGF21 siRNA-treated BM-MSCs at P4 (i, ii). (c) Representative images of SA- $\beta$-gal staining in control siRNA or FGF21 siRNA-treated BM-MSCs at P4 (i). Percentage of SA- $\beta$-gal-positive senescent cells in control siRNA or FGF21 siRNA-treated-BMMSCs at P4 (ii). (d) Representative images of Ki-67 staining in control siRNA or FGF21 siRNA-treated BM-MSCs at P4 (i). Percentage of Ki-67-positive senescent cells in control siRNA or FGF21 siRNA-treated BM-MSCs at P4 (ii). Data are expressed as mean \pm SEM with $n=$ 3 per group. ${ }^{* *} p<0.01$. Scale bar $=100 \mu \mathrm{m}$.

SA- $\beta$-gal activity (Figures 4(d) and 4(e)). Collectively, we conclude that FGF21 regulates the senescence of BM-MSCs via the mediation of mitochondrial dynamics.

\subsection{FGF21 Regulates Mitochondrial Dynamics by Mediating} AMPK Activation. Previous studies have demonstrated that AMPK regulates mitochondrial structure/function and dynamics [22, 23]. We attempted to determine whether FGF21 regulates mitochondrial dynamics via mediation of AMPK. First, we examined AMPK activation in different passages of MSCs. Western blotting showed that AMPK phosphorylation ( $\mathrm{p}$-AMPK) was gradually reduced in MSCs during consecutive passaging expansion (Figure 5(a), i and ii). To determine whether AMPK activation is responsible for 
i
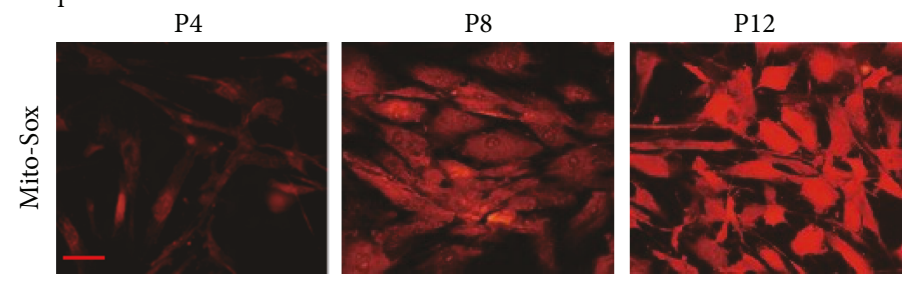

(a)

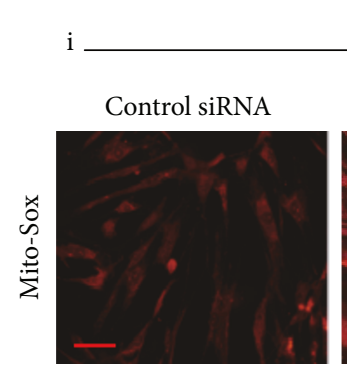

P4

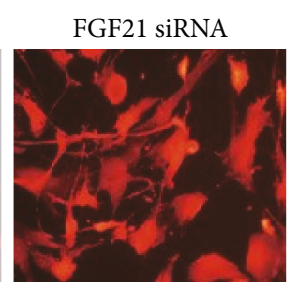

\section{FGF21 siRNA+}

MitoTEMPO

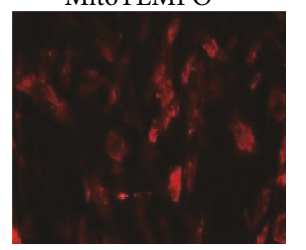

ii

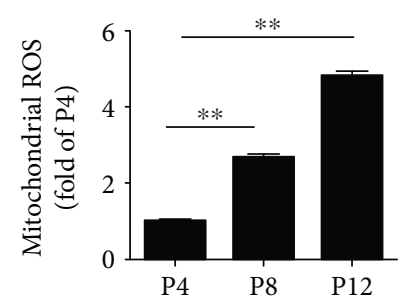

ii

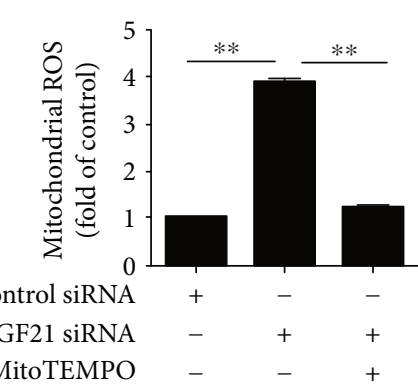

(b)

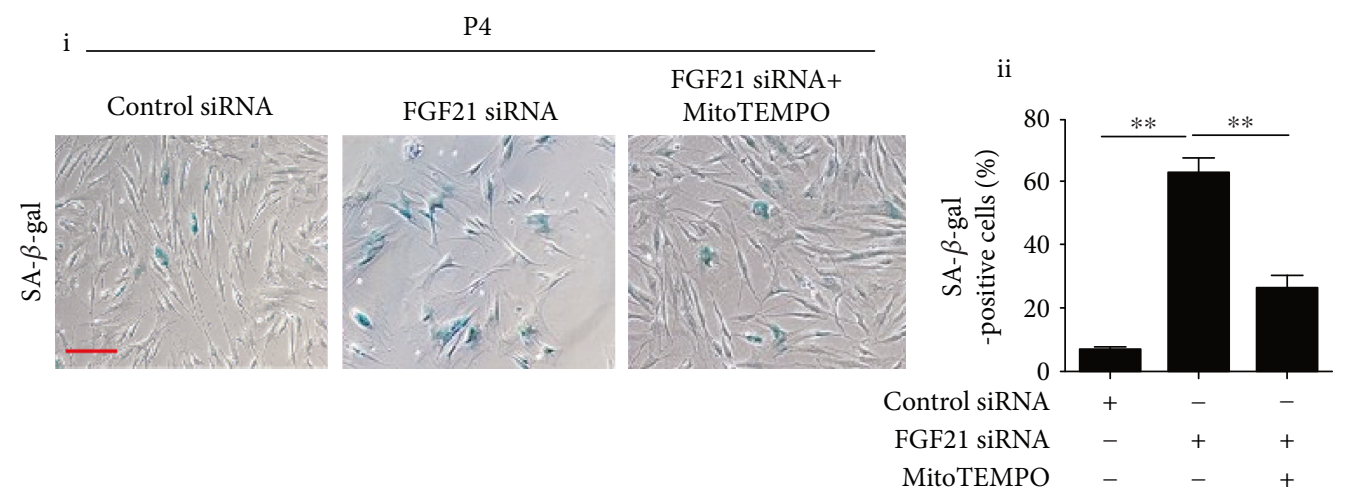

(c)

Figure 3: Silencing FGF21 induces MSC senescence via ROS generation. (a) Representative images of Mito-Sox staining in BM-MSCs at P4, P8, and P12 (i). Quantitative analysis of ROS generation in BM-MSCs at P4, P8, and P12 (ii). (b) Representative images of Mito-Sox staining in BM-MSCs at P4 from the control siRNA or FGF21 siRNA or FGF21 siRNA+MitoTEMPO-treated group (i). Quantitative analysis of ROS generation in control siRNA or FGF21 siRNA or FGF21 siRNA+MitoTEMPO-treated BM-MSCs at P4 (ii). (c) Representative images of SA$\beta$-gal staining in BM-MSCs at P4 from the control siRNA or FGF21 siRNA or FGF21 siRNA+MitoTEMPO-treated group (i). Percentage of SA- $\beta$-gal-positive senescent cells in control siRNA or FGF21 siRNA or FGF21 siRNA+MitoTEMPO-treated BM-MSCs at P4 (ii). Data are expressed as mean \pm SEM with $n=3$ per group. ${ }^{* *} p<0.01$. Scale bar $=100 \mu \mathrm{m}$.

mitochondrial dynamics, we treated P4 BM-MSCs with compound $\mathrm{C}$, an AMPK inhibitor. Compound $\mathrm{C}$ treatment significantly upregulated Mnf2 protein but downregulated p-Drp1 (Figure S3A-i, ii). Furthermore, compound C treatment significantly enhanced the senescence of MSCs at P4 (Figure S3B-i, ii). These results suggest that the AMPK pathway may participate in regulation of MSC senescence by mediating mitochondrial dynamics. Subsequently, we examined whether FGF21 mediates mitochondrial dynamics by regulating AMPK. Western blotting analysis showed that silencing FGF21 with FGF21 siRNA reduced p-AMPK and p-Drp1 and upregulated the Mfn2 level (Figure 5(b)) in P4 BM-MSCs. Nonetheless, the AMPK activator, AICAR, enhanced the reduced p-AMPK and p-Drp1 and decreased the upregulated Mfn2 in FGF21 siRNA-treated MSCs (Figure 5(b)). Furthermore, AICAR abrogated FGF21 silenceinduced MSC senescence (Figure 5(c)). To further verify whether FGF21 regulates mitochondrial dynamics by activating AMPK, we overexpressed FGF21 in P12 BMMSCs. Western blotting showed that overexpressed FGF21 in P12 BM-MSCs greatly enhanced the protein level of pAMPK and p-Drp1 but reduced Mfn2 (Figure S4). Furthermore, these effects were partly reversed by the AMPK inhibitor, compound C (Comp C) (Figure S4). These results indicate that FGF21 regulates mitochondrial dynamics via the AMPK signaling pathway and in turn mediates cell senescence. 

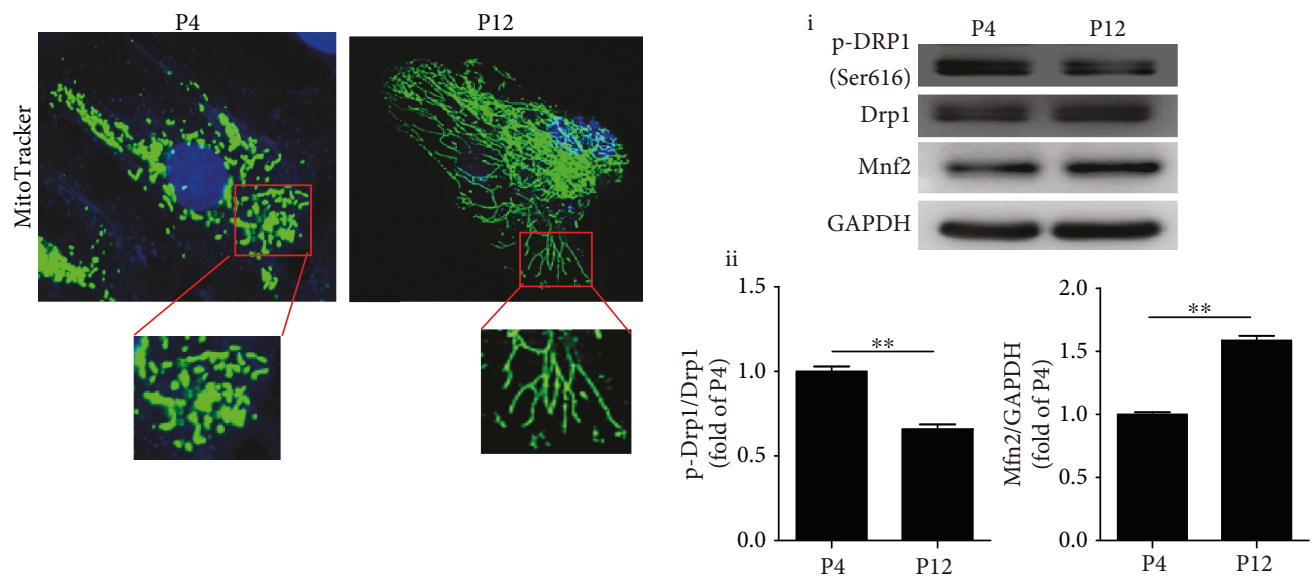

(a)

(b)
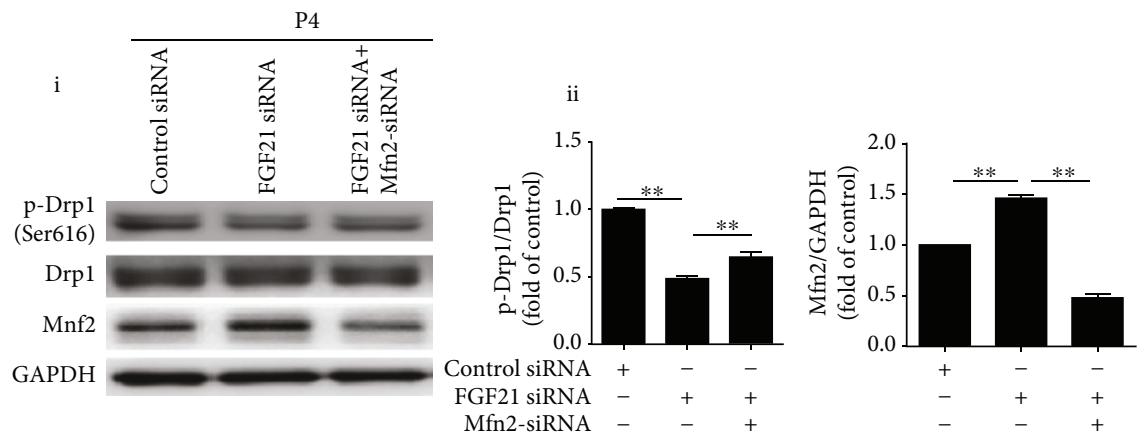

(c)
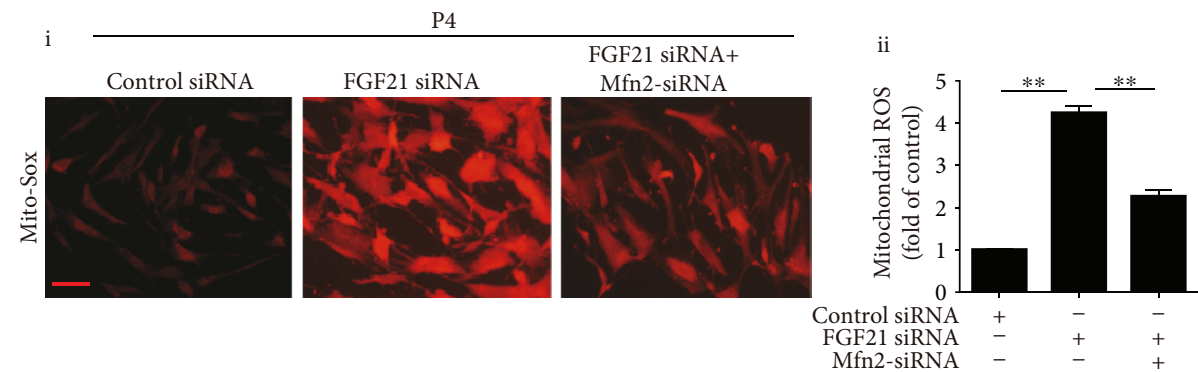

(d)

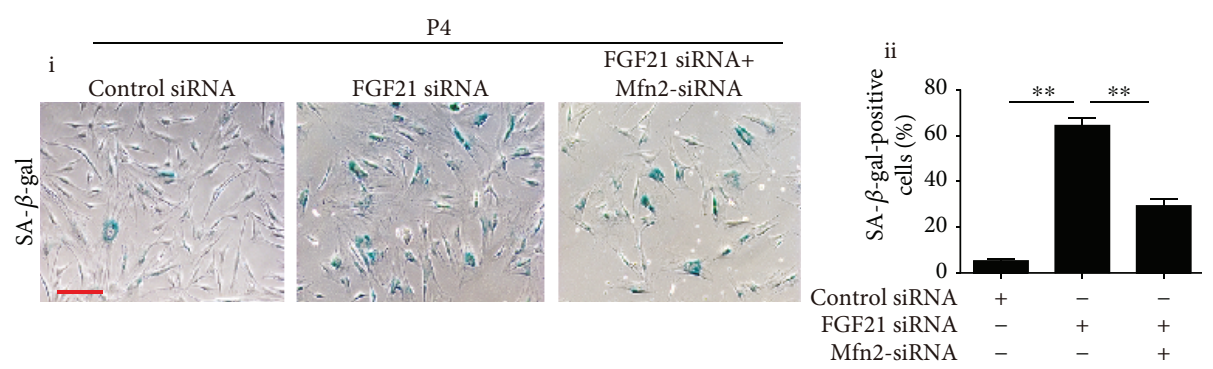

(e)

FIGURE 4: Silencing FGF21 induces ROS generation via regulation of mitochondrial dynamics. (a) Representative images of MitoTracker staining in BM-MSCs at P4 and P12. (b) Western blotting and quantitative analysis of the levels of p-Drp1/Drp1 and Mfn2 proteins in BM-MSCs at P4 and P12 (i, ii). (c) Western blotting and quantitative analysis of the level of p-Drp1/Drp1 and Mfn2 proteins in control siRNA or FGF21 siRNA or FGF21 siRNA+Mfn2 siRNA-treated BM-MSCs at P4 (i, ii). (d) Representative images of Mito-Sox staining in BM-MSCs at P4 from the control siRNA or FGF21 siRNA or FGF21 siRNA+Mfn2 siRNA-treated group (i). Quantitative analysis of the ROS generation in control siRNA or FGF21 siRNA or FGF21 siRNA+Mfn2 siRNA-treated BM-MSCs at P4 (ii). (e) Representative images of SA- $\beta$-gal staining in BM-MSCs at P4 from the control siRNA or FGF21 siRNA or FGF21 siRNA+Mfn2 siRNA-treated group (i). Percentage of SA- $\beta$-gal-positive senescent cells in control siRNA or FGF21 siRNA or FGF21 siRNA+Mfn2 siRNA-treated BM-MSCs at P4 (ii). Data are expressed as mean \pm SEM with $n=3$ per group. ${ }^{* *} p<0.01$. Scale bar $=100 \mu \mathrm{m}$. 

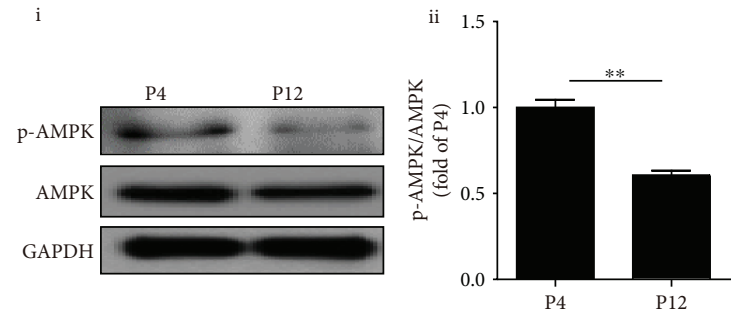

(a)
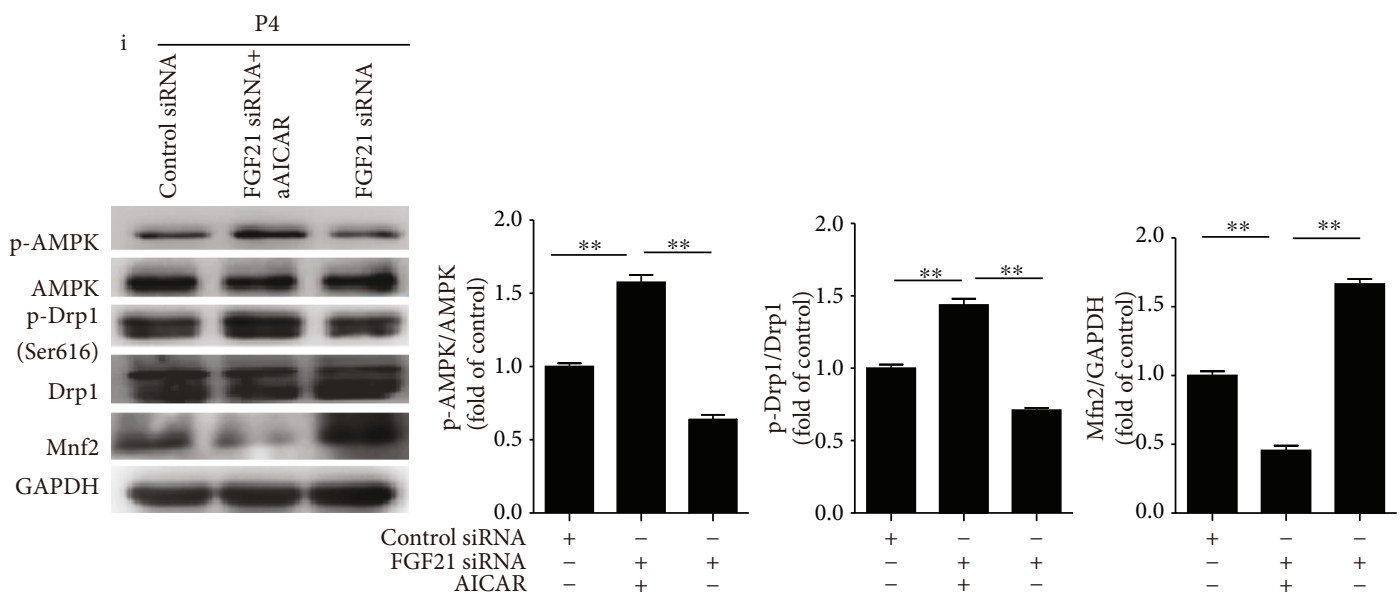

(b)
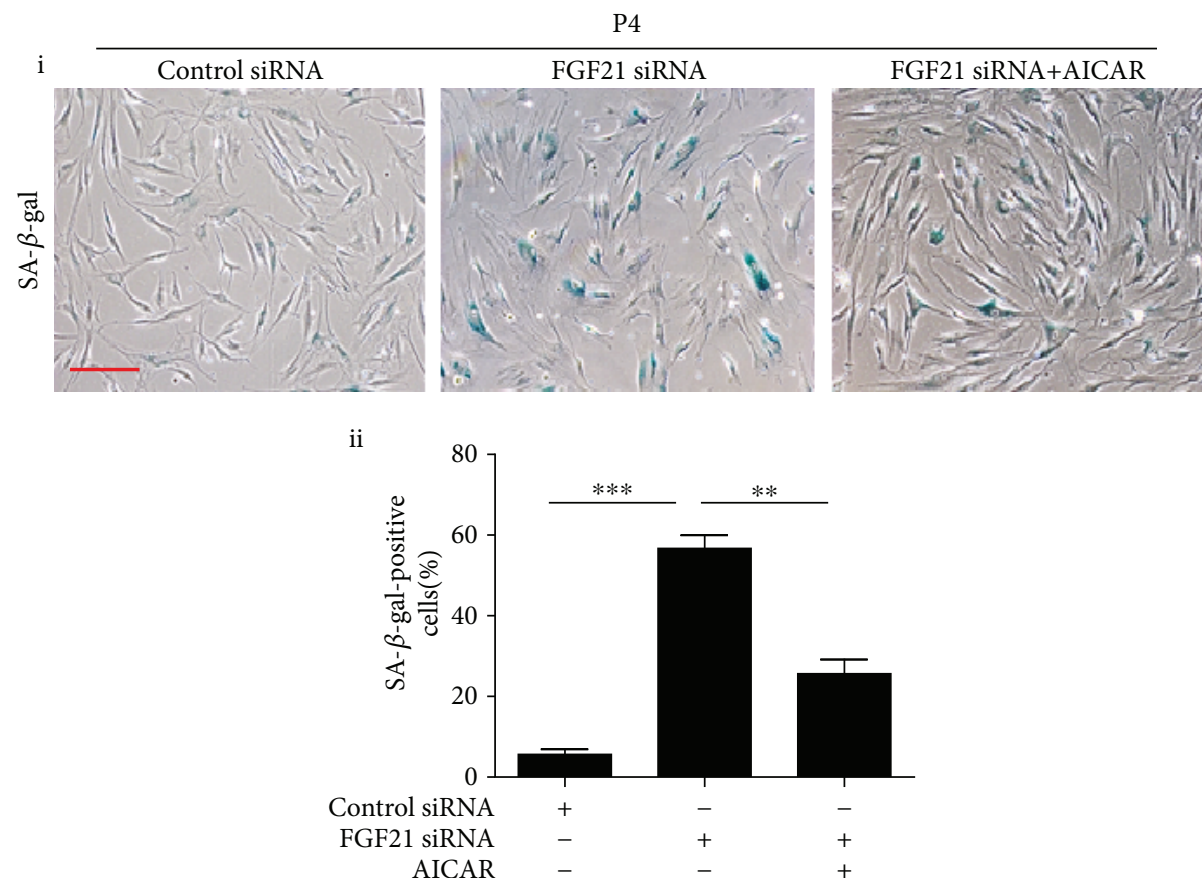

(c)

FIGURE 5: FGF21 regulates mitochondrial dynamics via mediation of AMPK activation. (a) Western blotting and quantitative analysis of the level of p-AMPK in BM-MSCs at P4 and P12 (i, ii). (b) Western blotting and quantitative analysis of the level of p-AMPK, p-Drp1, and Mfn2 in BM-MSCs at P4 from the control siRNA or FGF21 siRNA or FGF21 siRNA+AICAR-treated group (i, ii). (c) Representative images of SA$\beta$-gal staining in BM-MSCs at P4 from the control siRNA or FGF21 siRNA or FGF21 siRNA+AICAR-treated group (i). Percentage of SA- $\beta$ gal-positive senescent cells in control siRNA or FGF21 siRNA or FGF21 siRNA+AICAR-treated BM-MSCs at P4 (ii). Data are expressed as mean \pm SEM with $n=3$ per group. ${ }^{*} p<0.05,{ }^{* *} p<0.01$. Scale bar $=100 \mu \mathrm{m}$. 
3.6. Overexpression of FGF21 in Aged MSCs Attenuates Cell Senescence. To determine whether FGF21 mediates the physiological senescence of MSCs, we isolated MSCs from young and aged donors and examined the cellular senescence. Compared with young MSCs, aged MSCs demonstrated a decreased level of FGF21 and increased protein level of p53 and p21 as well as activity of SA- $\beta$-gal (Figures 6(a) and $6(\mathrm{~b}))$. On the contrary, overexpression of FGF21 in aged MSCs dramatically reduced the activity of SA- $\beta$-gal (Figure $6(\mathrm{c})$, i and ii). Furthermore, overexpression of FGF21 enhanced the level of FGF21 and reduced the levels of p53 and p21 proteins in aged MSCs (Figure 6(d), i and ii). These observations support the notion that FGF21 also regulates the physiological senescence of MSCs.

\section{Discussion}

There are some major findings in the current study (Figure 6(e)). First, the level of FGF21 was significantly reduced in MSCs with increased passage and in MSCs isolated from aged donors. Second, disruption of mitochondrial dynamics and induced ROS production resulted in senescence of MSCs. Third, FGF21 mediated MSC senescence via regulation of mitochondrial dynamics. Last but not least, the AMPK signaling pathway was involved in FGF21 regulation of mitochondrial dynamics.

Although MSC-based therapy has shown promising therapeutic potential in a variety of disorders in preclinical studies and clinical trials [24-26], MSCs can easily become senescent even under standard culture conditions as the passage increases in vitro, thus reducing their beneficial effects $[27,28]$. Senescent MSCs can release a variety of factors including inflammatory cytokines, termed senescenceassociated secretory phenotype (SASP). These factors further exacerbate cellular senescence and reduce the function of MSCs; thus, a vicious circle begins [29]. The senescent MSCs exhibit growth arrest, enlarged cell size, increased lysosomal content characterized by SA- $\beta$-gal, and lower proliferative capacity [30]. The activity of p53, by inducing a downstream gene p21, is positively correlated with cellular senescence [31]. In this study, we showed that BM-MSCs developed into the senescent phenotype during consecutive passaging expansion as evidenced by an increased cell size, a lower level of Ki-67, enhanced SA- $\beta$-gal positivity, and elevation of the p53 and p21 level.

Since BM-MSCs show replicative senescence in long-term culture, elucidating the potential mechanisms that underlie MSC senescence may help provide novel therapeutic strategies to delay senescence. An increasing number of studies have shown that FGF21 is a very important regulator in controlling longevity. Compared with wild-type mice, transgenic mice that overexpress FGF21 live longer with no change in food intake [32]. Furthermore, FGF21 reduces immune senescence by inhibiting age-related thymic involution [33]. These observations suggest that FGF21 has a strong antiaging capacity. Indeed, in the current study, we found that MSCs displayed a decreased level of FGF21 that was accompanied by an increased level of p53 and p21 during the passaging process, indicating that FGF21 may participate in the regulation of the replicative senescence of MSCs. Furthermore, knockdown of FGF21 using siRNA in the early passage of MSCs reinforced the senescence. On the contrary, overexpression of FGF21 in aged MSCs ameliorated the senescence. These findings are further evidence that FGF21 regulates both the physiological and replicative senescence of MSCs.

Despite thorough investigation, the molecular network underlying MSC senescence is not fully understood. Several mechanisms including telomere shortening, autophagy, and especially increased ROS have been well documented in MSC senescence [6, 34, 35]. Monocyte chemoattractant protein-1 (MCP-1) reinforces the senescence of MSCs derived from umbilical cord blood via activation of the ROS-p38-MAPK-p53/p21 signaling pathway [36]. In accordance with previous studies [1,37], our results also showed that the senescent MSCs (P8, P12) exhibited an increased level of ROS. Knockdown of FGF21 in the early passage of MSCs enhanced ROS generation whereas ROS scavengers significantly prevented the senescence, indicating that knockdown of FGF21 induces senescence of MSCs by inducing ROS generation. ROS production is mainly mediated by the imbalance between mitochondrial fusion and fission [16, 38]. Depletion of protein disulfide isomerase A1 (PDIA1) promotes mitochondrial fission and elevates ROS generation and finally induces endothelial cell senescence [39]. In contrast, downregulation of Drp1 inducing mitochondrial fusion contributes to endothelial cell senescence by enhancing ROS production [40]. Moreover, the senescent MSCs isolated from human adipose tissue exhibit increased mitochondrial fusion and enhanced ROS production [37]. These contrary results may be due to the different types of cells and different stresses that caused cellular senescence. In this study, we found enhanced mitochondrial fusion as evidenced by elongated mitochondria and enhanced Mfn2 and decreased pDrp1 protein P12 BM-MSCs compared with P4 BM-MSCs. Inhibition of mitochondrial fission using Mdivi-1 dramatically enhanced mitochondrial ROS production and increased MSC senescence. More importantly, we also showed that knockdown of FGF21 in the early passage of MSCs greatly elevated mitochondrial fusion as evidenced by the enhanced Mfn2 and decreased p-Drp1. Nevertheless, knockdown of Mfn2 reversed FGF21 knockdown-induced mitochondrial fusion and senescence of MSCs. Based on the above results, we conclude that FGF21 mediates MSC senescence via regulation of mitochondrial dynamics.

Adenosine monophosphate-activated protein kinase (AMPK) is a conserved, redox-activated master regulator of cell metabolism. Recently, an increasing number of studies have shown that AMPK signaling is essential to maintain mitochondrial dynamics and function [41, 42]. AMPK activation enhances mitochondrial fission via phosphorylation of mitochondrial fission factor (MFF) and ULK1 [43, 44]. FGF21 is closely related to AMPK activation [45]. FGF21 extends the mammalian lifespan via activation of AMPK [9]. Moreover, the protective effects of FGF21 on cardiomyopathy have been attributed to activation of AMPK [46, 47]. Given the relationship between FGF21 and AMPK, we next addressed whether FGF21 regulation of mitochondrial dynamics is via mediation of AMPK activation. We showed 

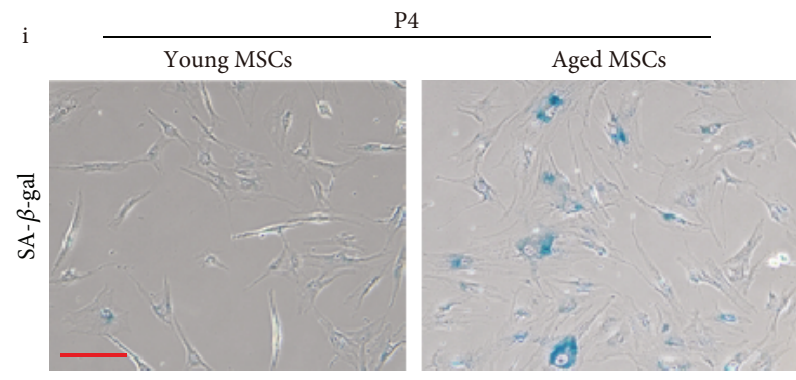

(a)

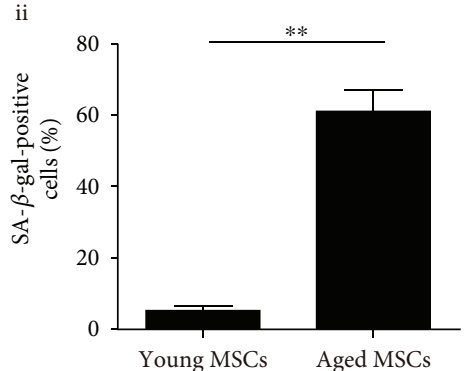

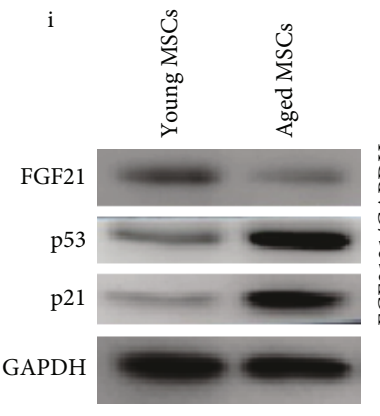

i

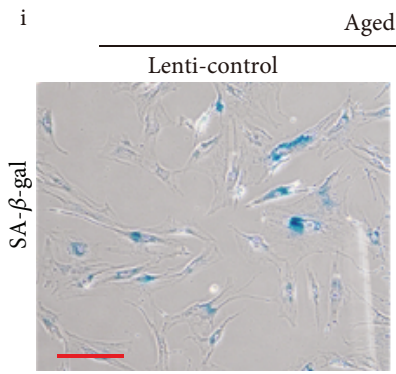

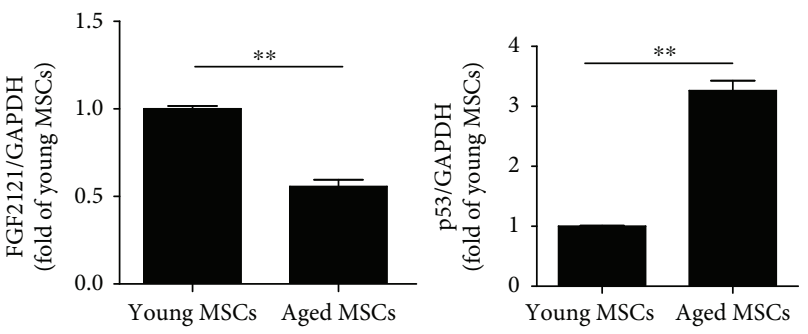

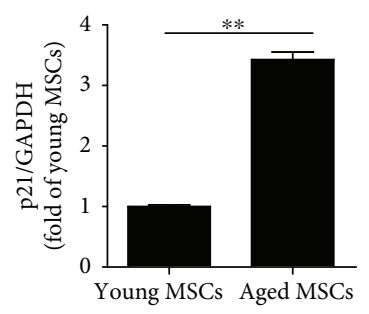

(b)

Lenti-FGF21

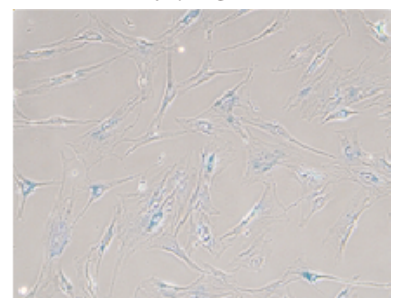

(c)
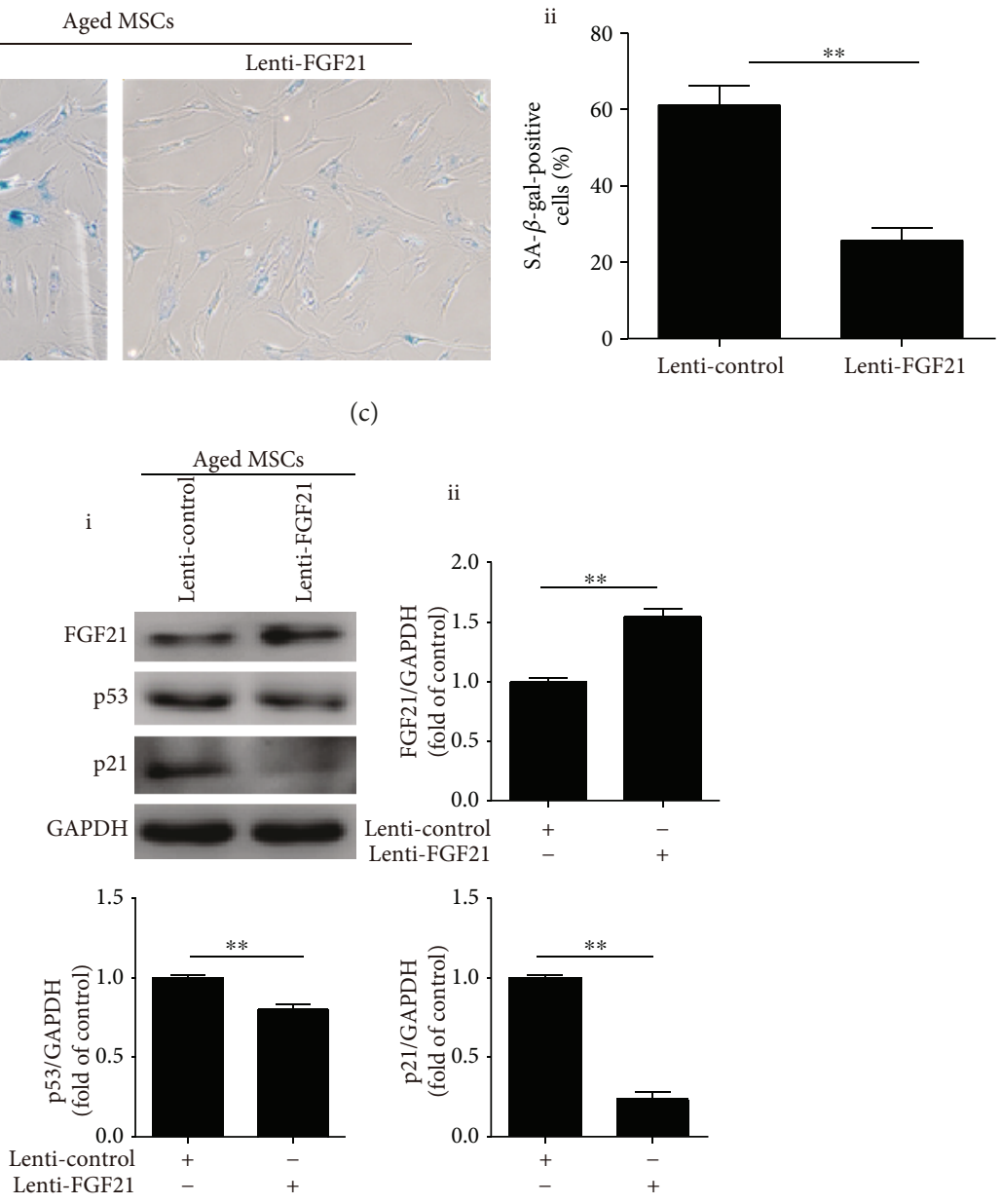

(d)

Figure 6: Continued. 

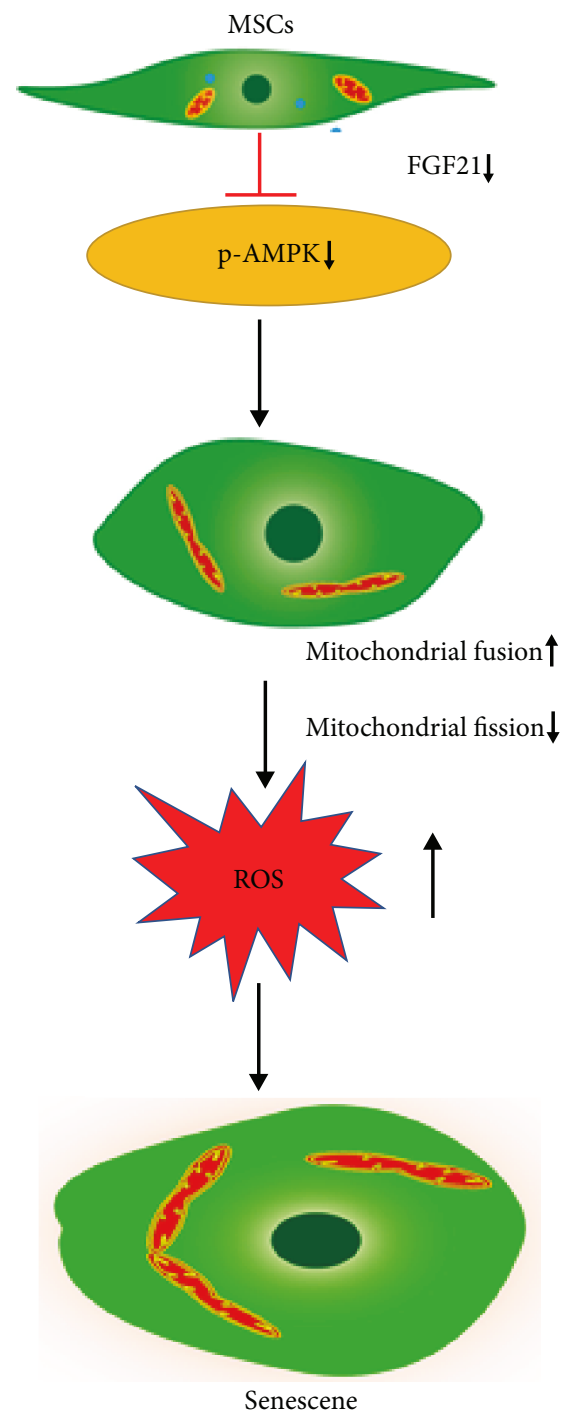

(e)

Figure 6: Overexpression of FGF21 in aged MSCs reduces cell senescence. (a) Representative images of SA- $\beta$-gal staining in BM-MSCs isolated from young and aged donors (i). Percentage of SA- $\beta$-gal-positive senescent cells in young MSCs and aged MSCs (ii). (b) Western blotting and quantitative analysis of the level of FGF21, p53, and p21 in young MSCs and aged MSCs (i, ii). (c) Representative images of SA- $\beta$-gal staining in aged BM-MSCs transfected with control lentivirus or FGF21 lentivirus (i). Percentage of SA- $\beta$-gal-positive senescent cells in aged BM-MSCs transfected with control lentivirus or FGF21 lentivirus (ii). (d) Western blotting and quantitative analysis of the level of FGF21, p53, and p21 in aged BM-MSCs transfected with control lentivirus or FGF21 lentivirus (i, ii). (e) The proposed mechanisms involved in FGF21 mediates MSC senescence via regulation of mitochondrial dynamics. Data are expressed as mean \pm SEM with $n=3$ per group. ${ }^{* *} p<0.01$. Scale bar $=100 \mu \mathrm{m}$.

that knockdown of FGF21 significantly reduced AMPK activation and enhanced mitochondrial fusion. In contrast, treatment with AICAR abrogated these effects and reduced knockdown of FGF21-induced cellular senescence.

Several limitations of the current study need to be highlighted. First, we used siRNA to knock down FGF21. It would better illustrate the role of FGF21 in the regulation of cell senescence if we had used Crispr/Cas9 to knock out FGF21. Second, the potential role of telomere shortening or autophagy in FGF21-mediated MSC senescence was not studied. Third, our study used only BM-MSCs. Whether these findings can be applied to MSCs derived from other tissues such as adipose and umbilical cord remains to be investigated. Last but not least, the mechanisms underlying FGF21 regulating the AMPK signal pathway still remain unclear.

\section{Conclusion}

In summary, our study demonstrates that FGF21 regulates the senescence of MSCs via mediation of mitochondrial dynamics and ROS production in an AMPK-dependent manner. FGF21 may be a promising target for rejuvenating the cellular senescence of MSCs. 


\section{Data Availability}

All data used in the current study are available from the corresponding author upon request.

\section{Conflicts of Interest}

The authors have no conflicts of interest to declare.

\section{Authors' Contributions}

Xin Li and Yimei Hong contributed equally to this study.

\section{Acknowledgments}

This research was in part supported by a grant from the National Natural Science Foundation of China (No. 81700259 to Y. Zhang; Nos. 81871599 and 81671882 to X. Li), the Guangdong Provincial People's Hospital Grant for Talent Introduction (No. Y012018144 to Y. Zhang), the Natural Science Foundation of Guangdong Province of China (2016A030311039 to X. Li), and the Science and Technology Foundation of Guangdong Province of China (2015A020212012 and 2017A020224012 to X. Li).

\section{Supplementary Materials}

Figure S1: the levels of mitochondrial dynamics-related proteins in BM-MSCs at P4 and P12. Figure S2: inhibition of mitochondrial fission induces cell senescence. Figure S3: inhibition of AMPK induces cell senescence via regulating mitochondrial dynamics. Figure S4: overexpression of FGF21 regulates mitochondrial dynamics via activating AMPK in P12 BM-MSCs. (Supplementary Materials)

\section{References}

[1] J. C. Estrada, Y. Torres, A. Benguría et al., "Human mesenchymal stem cell-replicative senescence and oxidative stress are closely linked to aneuploidy," Cell Death \& Disease, vol. 4, no. 6, article e691, 2013.

[2] M. Kapetanou, N. Chondrogianni, S. Petrakis, G. Koliakos, and E. S. Gonos, "Proteasome activation enhances stemness and lifespan of human mesenchymal stem cells," Free Radical Biology \& Medicine, vol. 103, pp. 226-235, 2017.

[3] N. Ridzuan, A. Al Abbar, W. K. Yip, M. Maqbool, and R. Ramasamy, "Characterization and expression of senescence marker in prolonged passages of rat bone marrow-derived mesenchymal stem cells," Stem Cells International, vol. 2016, Article ID 8487264, 14 pages, 2016.

[4] W. L. Fu, J. Li, G. Chen, Q. Li, X. Tang, and C. H. Zhang, "Mesenchymal stem cells derived from peripheral blood retain their pluripotency, but undergo senescence during long-term culture," Tissue Engineering Part C, Methods, vol. 21, no. 10, pp. 1088-1097, 2015.

[5] R. A. Denu, "SIRT3 enhances mesenchymal stem cell longevity and differentiation," Oxidative Medicine and Cellular Longevity, vol. 2017, Article ID 5841716, 11 pages, 2017.

[6] Y. Ma, M. Qi, Y. An et al., "Autophagy controls mesenchymal stem cell properties and senescence during bone aging," Aging Cell, vol. 17, no. 1, 2018.
[7] J. C. Sepúlveda, M. Tomé, M. E. Fernández et al., "Cell senescence abrogates the therapeutic potential of human mesenchymal stem cells in the lethal endotoxemia model," Stem Cells, vol. 32, no. 7, pp. 1865-1877, 2014.

[8] T. Inagaki, P. Dutchak, G. Zhao et al., "Endocrine regulation of the fasting response by PPAR $\alpha$-mediated induction of fibroblast growth factor 21," Cell Metabolism, vol. 5, no. 6, pp. 415-425, 2007.

[9] A. Salminen, A. Kauppinen, and K. Kaarniranta, "FGF21 activates AMPK signaling: impact on metabolic regulation and the aging process," Journal of Molecular Medicine, vol. 95, no. 2, pp. 123-131, 2017.

[10] J. Yan, J. Wang, H. Huang et al., "Fibroblast growth factor 21 delayed endothelial replicative senescence and protected cells from $\mathrm{H}_{2} \mathrm{O}_{2}$-induced premature senescence through SIRT1," American Journal of Translational Research, vol. 9, no. 10, pp. 4492-4501, 2017.

[11] X. M. Wang, H. Xiao, L. L. Liu, D. Cheng, X. J. Li, and L. Y. Si, "FGF21 represses cerebrovascular aging via improving mitochondrial biogenesis and inhibiting p53 signaling pathway in an AMPK-dependent manner," Experimental Cell Research, vol. 346, no. 2, pp. 147-156, 2016.

[12] M. Hochane, V. Trichet, C. Pecqueur et al., "Low-dose pesticide mixture induces senescence in normal mesenchymal stem cells (MSC) and promotes tumorigenic phenotype in premalignant MSC," Stem Cells, vol. 35, no. 3, pp. 800-811, 2017.

[13] P.-M. Chen, C. H. Lin, N. T. Li et al., "c-Maf regulates pluripotency genes, proliferation/self-renewal, and lineage commitment in ROS-mediated senescence of human mesenchymal stem cells," Oncotarget, vol. 6, no. 34, pp. 35404-35418, 2015.

[14] L. Zhou, R. Li, C. Liu et al., "Foxo3a inhibits mitochondrial fission and protects against doxorubicin-induced cardiotoxicity by suppressing MIEF2," Free Radical Biology \& Medicine, vol. 104, pp. 360-370, 2017.

[15] D. Zhang, Y. Liu, Y. Tang et al., "Increased mitochondrial fission is critical for hypoxia-induced pancreatic beta cell death," PLoS One, vol. 13, no. 5, article e0197266, 2018.

[16] J. Ježek, K. F. Cooper, and R. Strich, "Reactive oxygen species and mitochondrial dynamics: the yin and yang of mitochondrial dysfunction and cancer progression," Antioxidants, vol. 7, no. 1, 2018.

[17] D. Q. Tan and T. Suda, "Reactive oxygen species and mitochondrial homeostasis as regulators of stem cell fate and function," Antioxidants \& Redox Signaling, vol. 29, no. 2, pp. 149168, 2018.

[18] P. Martin-Maestro, R. Gargini, E. Garcia, G. Perry, J. Avila, and V. Garcia-Escudero, "Slower dynamics and aged mitochondria in sporadic Alzheimer's disease," Oxidative Medicine and Cellular Longevity, vol. 2017, Article ID 9302761, 14 pages, 2017.

[19] M. Song, A. Franco, J. A. Fleischer, L. Zhang, and G. W. Dorn II, "Abrogating mitochondrial dynamics in mouse hearts accelerates mitochondrial senescence," Cell Metabolism, vol. 26, no. 6, pp. 872-883.e5, 2017.

[20] M. Drefs, M. N. Thomas, M. Guba et al., "Modulation of glutathione hemostasis by inhibition of 12/15-lipoxygenase prevents ROS-mediated cell death after hepatic ischemia and reperfusion," Oxidative Medicine and Cellular Longevity, vol. 2017, Article ID 8325754, 12 pages, 2017.

[21] H. F. Song, S. He, S. H. Li et al., "Aged human multipotent mesenchymal stromal cells can be rejuvenated by neuron- 
derived neurotrophic factor and improve heart function after injury," JACC: Basic to Translational Science, vol. 2, no. 6, pp. 702-716, 2017.

[22] J. Zhang, Y. Wang, X. Liu, R. K. Dagda, and Y. Zhang, "How AMPK and PKA interplay to regulate mitochondrial function and survival in models of ischemia and diabetes," Oxidative Medicine and Cellular Longevity, vol. 2017, Article ID 4353510, 12 pages, 2017.

[23] Q. Wang, M. Zhang, G. Torres et al., "Metformin suppresses diabetes-accelerated atherosclerosis via the inhibition of Drp1-mediated mitochondrial fission," Diabetes, vol. 66, no. 1, pp. 193-205, 2017.

[24] Y. Zhang, Z. Yu, D. Jiang et al., "iPSC-MSCs with high intrinsic MIRO1 and sensitivity to TNF- $\alpha$ yield efficacious mitochondrial transfer to rescue anthracycline-induced cardiomyopathy," Stem Cell Reports, vol. 7, no. 4, pp. 749-763, 2016.

[25] X. Liang, Y. Ding, Y. Zhang et al., “Activation of NRG1-ERBB4 signaling potentiates mesenchymal stem cell-mediated myocardial repairs following myocardial infarction," Cell Death \& Disease, vol. 6, no. 5, article e1765, 2015.

[26] A. Saad, A. B. Dietz, S. M. S. Herrmann et al., “Autologous mesenchymal stem cells increase cortical perfusion in renovascular disease," Journal of the American Society of Nephrology, vol. 28, no. 9, pp. 2777-2785, 2017.

[27] T. Zhang, P. Wang, Y. Liu et al., "Overexpression of FOXQ1 enhances anti-senescence and migration effects of human umbilical cord mesenchymal stem cells in vitro and in vivo," Cell and Tissue Research, vol. 373, no. 2, pp. 379-393, 2018.

[28] J. Kim, Y. Kim, H. Choi et al., "Ubiquitin C decrement plays a pivotal role in replicative senescence of bone marrow mesenchymal stromal cells," Cell Death \& Disease, vol. 9, no. 2, p. 139, 2018.

[29] J. Y. Lee, K. R. Yu, B. C. Lee et al., “GATA4-dependent regulation of the secretory phenotype via MCP-1 underlies lamin Amediated human mesenchymal stem cell aging," Experimental \& Molecular Medicine, vol. 50, no. 5, p. 63, 2018.

[30] V. V. Lunyak, A. Amaro-Ortiz, and M. Gaur, "Mesenchymal stem cells secretory responses: senescence messaging secretome and immunomodulation perspective," Frontiers in Genetics, vol. 8, p. 220, 2017.

[31] C. Jiang, G. Liu, T. Luckhardt et al., "Serpine 1 induces alveolar type II cell senescence through activating p53-p21-Rb pathway in fibrotic lung disease," Aging Cell, vol. 16, no. 5, pp. 11141124, 2017.

[32] Y. Zhang, Y. Xie, E. D. Berglund et al., "The starvation hormone, fibroblast growth factor-21, extends lifespan in mice," Elife, vol. 1, article e00065, 2012.

[33] Y. H. Youm, T. L. Horvath, D. J. Mangelsdorf, S. A. Kliewer, and V. D. Dixit, "Prolongevity hormone FGF21 protects against immune senescence by delaying age-related thymic involution," Proceedings of the National Academy of Sciences of the United States of America, vol. 113, no. 4, pp. 1026-1031, 2016.

[34] M. Okada, H. W. Kim, K. Matsu-ura, Y. G. Wang, M. Xu, and M. Ashraf, "Abrogation of age-induced microRNA-195 rejuvenates the senescent mesenchymal stem cells by reactivating telomerase," Stem Cells, vol. 34, no. 1, pp. 148-159, 2016.

[35] R. Vono, E. Jover Garcia, G. Spinetti, and P. Madeddu, "Oxidative stress in mesenchymal stem cell senescence: regulation by coding and noncoding RNAs," Antioxidants \& Redox Signaling, vol. 29, no. 9, pp. 864-879, 2018.
[36] H. J. Jin, H. J. Lee, J. Heo et al., "Senescence-associated MCP-1 secretion is dependent on a decline in BMI1 in human mesenchymal stromal cells," Antioxidants \& Redox Signaling, vol. 24, no. 9, pp. 471-485, 2016.

[37] B. R. Stab II, L. Martinez, A. Grismaldo et al., "Mitochondrial functional changes characterization in young and senescent human adipose derived MSCs," Frontiers in Aging Neuroscience, vol. 8, p. 299, 2016.

[38] D. Maggiorani, N. Manzella, D. E. Edmondson et al., "Monoamine oxidases, oxidative stress, and altered mitochondrial dynamics in cardiac ageing," Oxidative Medicine and Cellular Longevity, vol. 2017, Article ID 3017947, 8 pages, 2017.

[39] Y. M. Kim, S. W. Youn, V. Sudhahar et al., "Redox regulation of mitochondrial fission protein Drp1 by protein disulfide isomerase limits endothelial senescence," Cell Reports, vol. 23, no. 12, pp. 3565-3578, 2018.

[40] J. R. Lin, W. L. Shen, C. Yan, and P. J. Gao, "Downregulation of dynamin-related protein 1 contributes to impaired autophagic flux and angiogenic function in senescent endothelial cells," Arteriosclerosis, Thrombosis, and Vascular Biology, vol. 35, no. 6, pp. 1413-1422, 2015.

[41] A. Sarre, J. Gabrielli, G. Vial, X. M. Leverve, and F. Assimacopoulos-Jeannet, "Reactive oxygen species are produced at low glucose and contribute to the activation of AMPK in insulin-secreting cells," Free Radical Biology \& Medicine, vol. 52, no. 1, pp. 142-150, 2012.

[42] R. Q. Xue, L. Sun, X. J. Yu, D. L. Li, and W. J. Zang, "Vagal nerve stimulation improves mitochondrial dynamics via an $\mathrm{M}_{3}$ receptor/CaMKK $\beta / A M P K$ pathway in isoproterenolinduced myocardial ischaemia," Journal of Cellular and Molecular Medicine, vol. 21, no. 1, pp. 58-71, 2017.

[43] D. F. Egan, D. B. Shackelford, M. M. Mihaylova et al., "Phosphorylation of ULK1 (hATG1) by AMP-activated protein kinase connects energy sensing to mitophagy," Science, vol. 331, no. 6016, pp. 456-461, 2011.

[44] E. Q. Toyama, S. Herzig, J. Courchet et al., "Metabolism. AMPactivated protein kinase mediates mitochondrial fission in response to energy stress," Science, vol. 351, no. 6270, pp. 275-281, 2016.

[45] X. M. Wang, S. S. Song, H. Xiao, P. Gao, X. J. Li, and L. Y. Si, "Fibroblast growth factor 21 protects against high glucose induced cellular damage and dysfunction of endothelial nitric-oxide synthase in endothelial cells," Cellular Physiology and Biochemistry, vol. 34, no. 3, pp. 658-671, 2014.

[46] H. Yang, A. Feng, S. Lin et al., "Fibroblast growth factor-21 prevents diabetic cardiomyopathy via AMPK-mediated antioxidation and lipid-lowering effects in the heart," Cell Death \& Disease, vol. 9, no. 2, p. 227, 2018.

[47] F. Wu, B. Wang, S. Zhang et al., "FGF21 ameliorates diabetic cardiomyopathy by activating the AMPK-paraoxonase 1 signaling axis in mice," Clinical Science, vol. 131, no. 15, pp. 18771893, 2017. 


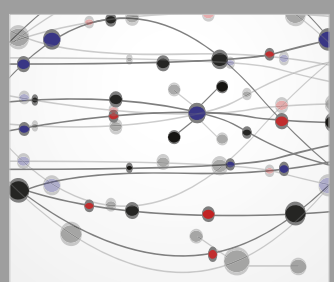

The Scientific World Journal
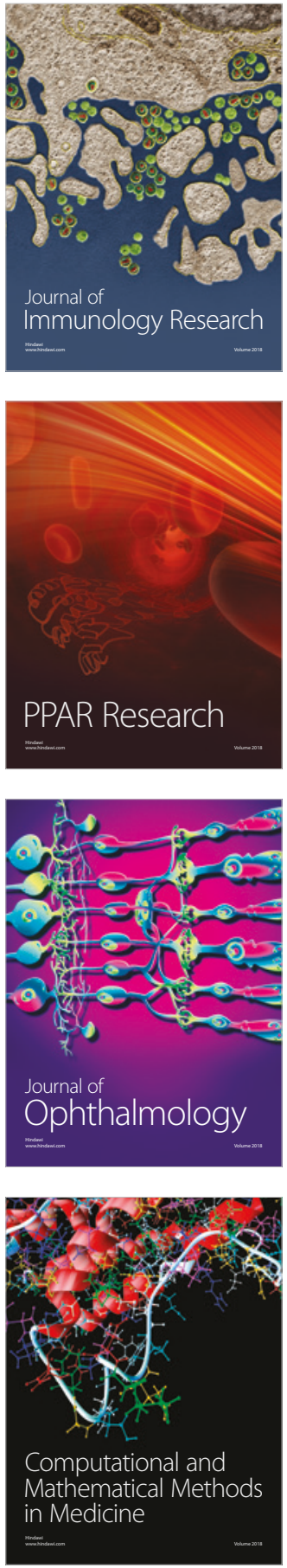

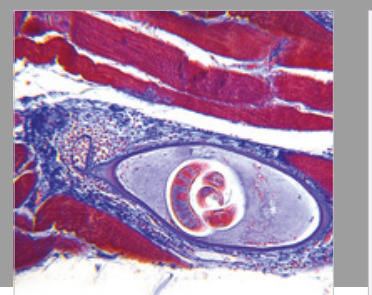

Gastroenterology Research and Practice

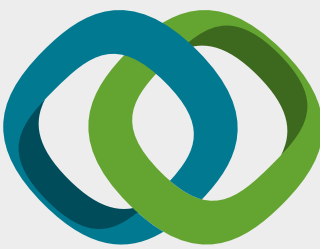

\section{Hindawi}

Submit your manuscripts at

www.hindawi.com
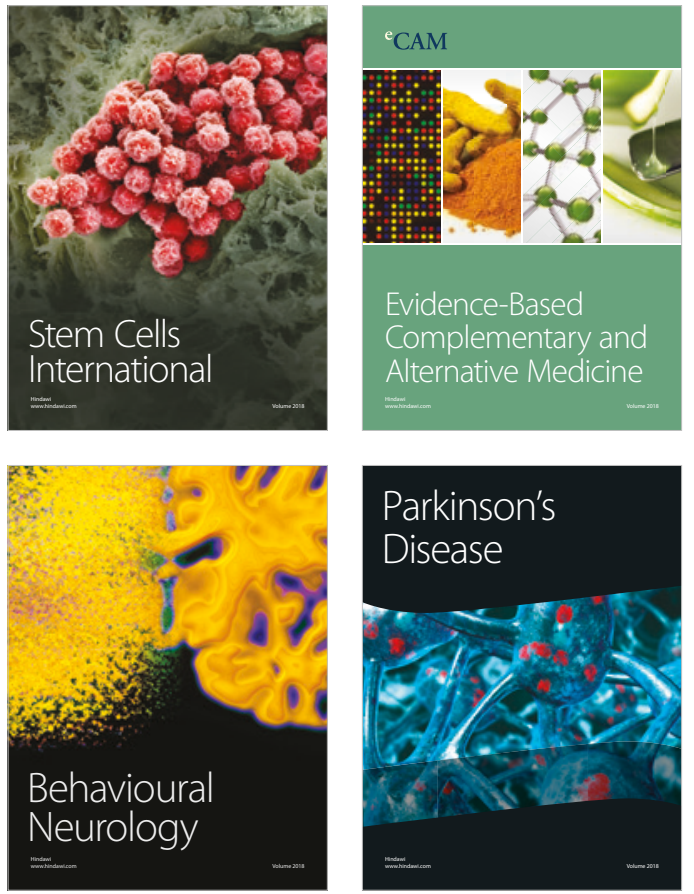

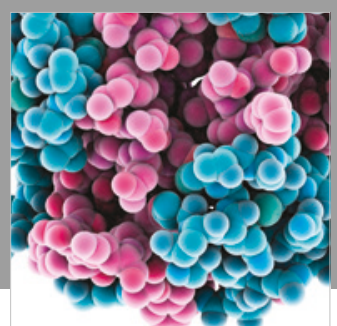

ournal of

Diabetes Research

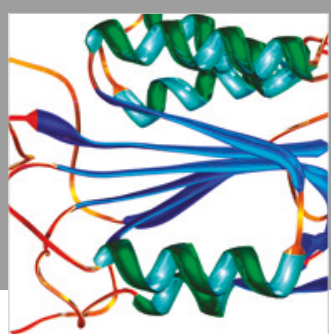

Disease Markers
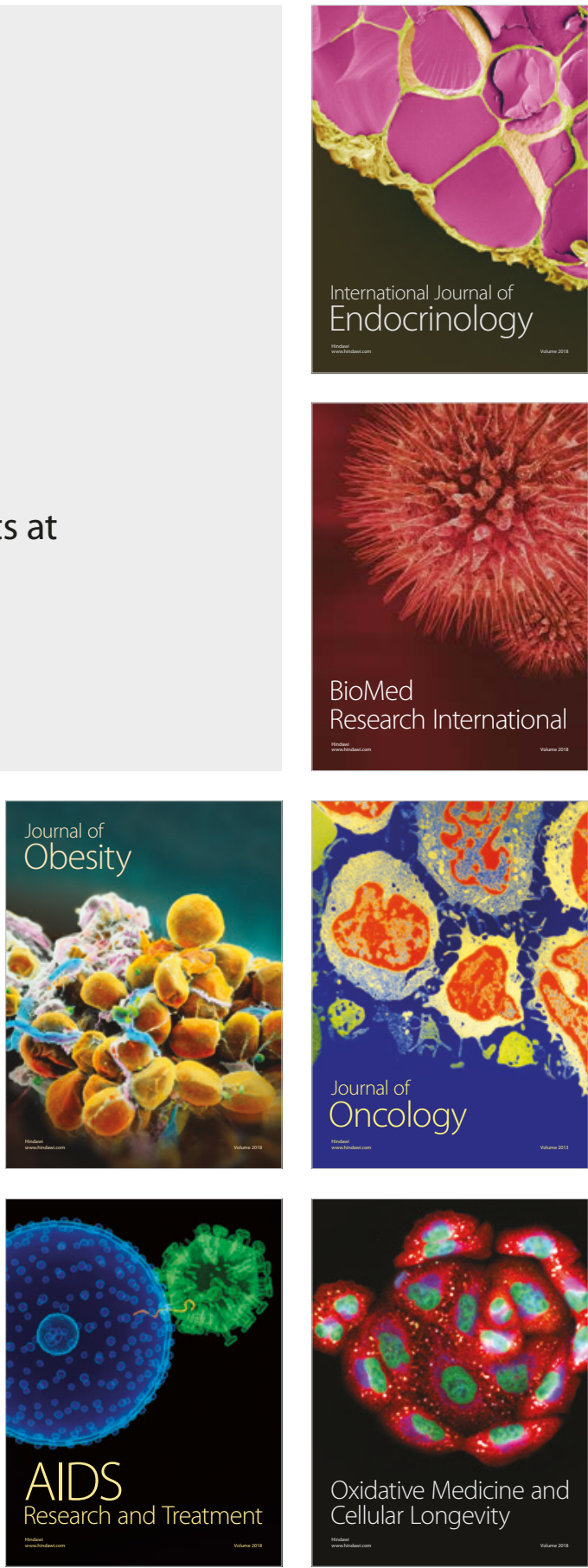